\title{
Violences coloniales et écriture de la transgression chez deux auteures ultra- marines : Déwé Görödé et Chantal Spitz
}

Audrey OGES, CNEP Université de la Nouvelle-Calédonie

\section{Introduction}

Édouard Glissant, dans son œuvre Poétique de la relation, déclare ceci : «Pendant une période historique de plus de deux siècles, l'identité affirmée des peuples devra se gagner contre les processus d'identification ou de néantisation déclenchés par ces envahisseurs. Si la nation en Occident est d'abord un 'contraire', l'identité pour les peuples colonisés sera en premier lieu un 'opposé à', c'est-à-dire au principe une limitation. Le vrai travail de la décolonisation aura été d'outrepasser cette limite» (29). Comme l'indique cet auteur, les littératures de pays anciennement colonisés, ou «postcoloniales », développent un mode d'expression original qui « outrepasse » des limites. La notion de transgression renvoie au non-respect de règles, de codes, en sous-entendant qu'il existe une norme à respecter. Cette norme incarne le modèle idéal, que tout sujet se doit de connaître et de suivre. Or, la transgression des interdits est partout dans cette littérature postcoloniale: transgressions politiques, discursives, sociales, etc. Ces auteurs se définissent dans un rapport de tension avec l'ordre établi : pour exister, ils choisissent de rejeter l'héritage colonial. Pourtant, paradoxalement, le français demeure la norme, la langue d'expression. Ces auteurs écrivent en français « sans coïncider tout à fait avec la nation française », comme le dit Patrick Sultan (58).

Ainsi, chez Déwé Görödé et Chantal Spitz, deux auteures respectivement kanak et polynésienne, on peut suivre des positions et propositions parfois ambiguës, liées à leur situation diglossique, biculturelle. Dans leurs œuvres, elles subvertissent les normes, les codes et les formes liées à la culture française, accédant ainsi à une liberté nouvelle. Il s'agit pour elles d'exprimer leurs souffrances, dans une position anti-coloniale, où elles résistent à l'inclusion dans l'État-nation. Ceci se traduit dans l'écriture, où le refus des normes est omniprésent : transgressions des genres, déconstruction des codes grammaticaux, recours à des formes linguistiques jugées «familières » ou «populaires» par les dictionnaires, expression de la révolte et de la violence, etc. Mais loin de vouloir être corrigées, ou de revenir à la «norme », elles assument cette nouvelle parole, et ce nouveau style d'écriture, qui est le leur.

On pourrait alors s'interroger sur la dimension constructive de la notion de 
transgression : est-elle simplement un non-respect des règles ou permet-elle d'ouvrir d'autres perspectives de lectures et d'expressions ? Peut-on, comme Michel Foucault, redéfinir cette notion en lui conférant un sens cette fois positif, c'est-à-dire une progression et non plus une régression? (29). Ainsi, on pourrait penser que ce dépassement de limites permet chez Görödé et Spitz l'écriture d'une réalité nouvelle, dans un sens positif pour le sujet. La transgression, qui flirte avec les interdits, acquerrait ici une dimension positive : celle que la géologie lui donnait, et qui est une invitation à dépasser, à se dépasser.

Nous étudierons tout d'abord la transgression que constitue le fait d'être pour Görödé et Spitz des écrivaines postcoloniales issues de territoires d'outre-mer; puis nous verrons l'écriture de ces deux femmes qui, engagées politiquement, ne respectent aucun code et écrivent dans une très grande liberté d'inspiration et de style; enfin, nous nous demanderons s'il n'existe pas chez elles une forme de « transgression joyeuse ».

\section{Présentation de la notion de transgression}

Görödé et Spitz ont choisi des modes d'expression polémiques à l'égard de l'ordre sous toutes ses formes: la notion de transgression est centrale dans leur œuvre. C'est pourquoi il nous faut la définir. Le terme de «transgression » vient du latin transgressus issu du verbe transgredior qui signifie, en verbe intransitif, "passer de l'autre côté, traverser, passer d'une chose à une autre ». Dès le troisième siècle, la transgressio signifie dans le latin chrétien «violation, péché, faute»: c'est la transgression, donc la violation des lois de l'Église (la transgression d'Adam et d'Eve). Ce terme est lié à la notion de culpabilité : « Situation d'une personne coupable ou tenue pour coupable, ou qui se sent à tort ou à raison coupable d'avoir transgressé une règle ». Au vingtième siècle, la transgression est ainsi définie : « action d'enfreindre un ordre, une loi, une obligation ». Cela renvoie au non-respect des lois, des règles. La transgression pose l'existence de limites, de normes à franchir. En somme, la transgression reste liée à la sphère du négatif, puisqu'en religion, les transgressions de l'interdit portent sur l'inceste ou le meurtre, et font que l'homme régresse en-deçà de l'humain. Dans un autre domaine, c'est la géologie qui s'est également approprié ce terme dès 1903, dans le sens de «l'envahissement d'un continent par la mer, dû à l'affaissement des terres émergées ou à une élévation générale du niveau des mers (ou des deux effets cumulés) ». Le terme acquiert alors un sens mélioratif, dans ce mouvement en avant de la mer qui franchit la limite du continent. En géologie, ce qui prédomine donc, c'est la notion d'empiéter sur quelque chose et donc de progresser vers. 


\section{La littérature postcoloniale comme transgression :}

Görödé et Spitz appartiennent à cette région du monde que l'on nomme le Pacifique, notamment les ex-TOM du Pacifique, puisque la première est kanak et la seconde est polynésienne. Cette littérature émergente d'écrivains autochtones a inspiré de nombreuses études, qui voient en elles des points communs, des œuvres non isolées. C'est ce que l'on appelle la littérature postcoloniale. L'histoire de la colonisation, puis de la décolonisation a fortement marqué les populations du Pacifique francophone, celles de la Nouvelle-Calédonie et de la Polynésie. Sultan définit la littérature du postcolonialisme comme « ce qui reste du colonial quand celui-ci a été, en son principe, aboli » (58). Il n'exclut donc pas que ces littératures peuvent s'inscrire encore aujourd'hui dans des situations coloniales. En d'autres termes, on se base sur un plan conceptuel, épistémologique et non pas chronologique. Il s'agit d'une situation d'écriture, et pas d'une situation dans l'axe du temps, qui se réfère à des pratiques de lecture et d'écriture intéressées par les phénomènes de domination.

L'écrivain postcolonial est avant tout anticolonial et refuse également d'appartenir à l'État-nation. Il se rapproche de la racine «anti», se positionnant dans un rejet des stéréotypes $\mathrm{du}$ discours colonial où il transgresse les limites imposées par les « envahisseurs ». En effet, la transgression des interdits est partout dans cette littérature. Leur écriture est l'expression d'une souffrance liée au colonialisme. Malgré cela, les deux auteurs ont choisi d'écrire en français, mais en transgressant ses codes, ses normes. L'histoire coloniale peut apparaître de manière explicite comme dans le poème de Görödé intitulé « À la tribu », rédigé dans les années 1970-72, bien avant que l'Accord de Nouméa ne reconnaisse en 1998 les zones d'ombre de la colonisation. L'écrivain décrit dans ce texte les visites des soldats français et d'autres représentants de l'ordre dans sa tribu, à Ponérihouen. Aucune date n'est évoquée, mais le registre est polémique : elle dénonce explicitement le comportement de ces hommes qui « traversent la tribu nous montrent un film/couchent avec filles et femmes/Ces jours-là la tribu est envahie »(Cendres, 33). On observe précisément ici le rejet de l'État-nation. D'ailleurs, le poème commence par ce premier vers : «À la tribu je suis chez moi » et se termine par le même vers, suivi de la conjonction de coordination «MAIS » mise en majuscules et suivie de points de suspension. Car d'autres " hôtes » indésirables suivent les soldats dans cette visite de la tribu : «le gouverneur entouré de gendarmes », puis « le gouverneur et sa suite » pour finir par « les forces de l'ordre ». Görödé décrit avec ironie le fait qu'on leur demande de crier à pleins poumons «Vive la France » et d'agiter « des petits drapeaux tricolores ». Mais la population reste docile et accepte même de préparer les «bougnas », de «faire la coutume », et de danser «le pilu des ancêtres »: la 
culture kanak semble ici folklorisée. L'auteur montre une population soumise à l'impérialisme français, qui se plie sans broncher aux demandes des visiteurs («se taire quand [le gouverneur] parle/applaudir quand il a parlé »). La dernière strophe rappelle au lecteur que, selon Görödé, la Nouvelle-Calédonie est encore sous le joug du colonialisme français : «Quand les forces de l'ordre sont à la tribu / nous ne leur disons pas / 'Vous êtes chez nous' / ce sont elles qui nous hurlent / 'Nous sommes chez nous' / 'Ici c'est la France' » (33).

De même, Spitz accuse l'état français de vouloir occulter les violences faites à son pays lors de la colonisation. Cette volonté de refaire l'histoire est accompagnée d'une volonté d'effacer des consciences et des mémoires ces épisodes malheureux : «L'histoire est avant tout un enjeu politique et cet enjeu est d'autant plus important dans les pays occupés qu'il s'agit d'éradiquer les mémoires des temps d'avant l'occupation / effacer jusqu'aux noms des femmes et hommes qui auraient pu ensemencer les mémoires et y enfanter le sentiment d'appartenir à une communauté digne et libre »(Litterama, 63). Dans ce pamphlet, les attaques sont directes: Spitz ne prend pas de voies détournées pour accuser l'Europe de vouloir réécrire l'histoire en imposant sa propre vision des événements, et en passant par le biais de l'école républicaine. Ainsi, les références à la colonisation et à ses acteurs sont explicites : l'inadéquation avec la nation française est clairement exprimée.

Pour autant, la notion de transgression ne concerne que la subversion littéraire, et non la transgression sociale. Ainsi, la littérature postcoloniale se caractérise de plusieurs manières : tout d'abord, il s'agit d'écrivains dont le pays a souffert du colonialisme, et cette souffrance se retrouve dans leurs écrits. Ils ne considèrent pas que leur pays n'est plus colonisé. L'histoire occupe une place importante dans leurs livres, et c'est une façon pour eux de faire entendre une voix originale : celle des minorités. Ils semblent d'ailleurs s'exprimer au nom d'une collectivité. La langue occupe ici une place primordiale puisque ces écrivains ont choisi d'écrire dans la langue des colons, en l'occurrence en français, même si chez certains auteurs comme Görödé le choix du français leur permet aussi d'être lus et compris par une plus grande majorité (compte-tenu du grand nombre de langues vernaculaires parlées en Nouvelle-Calédonie, le français reste la langue utilisée pour se faire comprendre). Enfin, leurs écrits sont contestataires et très souvent teintés de violence.

\section{3. Être un écrivain océanien francophone : une forme de transgression}

Görödé et Spitz sont deux femmes très engagées, toutes les deux indépendantistes. Elles habitent en Océanie, et sont très préoccupées de l'avenir de 
leurs pays, qui, pour elles, restent colonisés. En Nouvelle-Calédonie, la littérature, entendue comme un échange de biens symboliques s'effectuant par écrit, est récente, comme l'affirme François Bogliolo qui parle d'une littérature en émergence. Cela désigne une littérature nouvelle, originale, mais qui ne renie ni ses origines ni ses héritages. Alors que la littérature écrite calédonienne a débuté au dix-huitième siècle par des récits de voyage avec deux écrits fondateurs pré-coloniaux, ceux de James Cook et de La Billardière (botaniste français qui écrit le récit de voyage pour la recherche de l'expédition de La Pérouse en Océanie, dans laquelle il est placé sous les ordres de Bruny d'Entrecasteaux), on fait naître la littérature kanak contemporaine entre 1969 et 1985, avec la publication d'Histoire et psychologie du Mélanésien du prêtre Anova, qui sera édité de manière posthume. Si elle fut longtemps dans l'ombre du pouvoir des colonisateurs, la littérature kanak tend depuis quelques années à faire entendre sa voix à travers l'écriture. Avec des auteurs comme Pierre Gope, dramaturge, Jean-Marie Tjibaou, homme politique et essayiste, ou Görödé, poète et romancière, la littérature kanak existe en s'appropriant des genres occidentaux. Comme l'a montré Hamid Mokaddem, elle est fortement marquée par la reformulation de la littérature orale kanak. Görödé, qui a publié son premier recueil de poèmes Sous les cendres des conques en 1985, est la seule femme écrivain kanak.

L'apparition d'une littérature polynésienne est également récente. On peut en dater les débuts dans les années soixante-dix. À cette époque, la Polynésie française se voit octroyer le statut de territoire autonome au sein de la République, avec une langue officielle, le français, et une langue autonome, le reo ma'ohi. Jusqu'à la fin des années soixante, la «littérature polynésienne », de Bougainville à Bernard Moitessier, se compose exclusivement d'écrits de voyageurs, de scientifiques et de missionnaires. En 1962, l'installation du Centre d'essais du Pacifique provoque un courant d'intérêt qui se traduit par une augmentation du nombre d'écrits sur la Polynésie, mais non par des Polynésiens. Le renouveau culturel $m a$ 'ohi date des années soixante-dix. De jeunes étudiants militent pour la reconnaissance du patrimoine culturel polynésien, et le font le plus souvent en français. Henri Hiro est considéré comme un précurseur et une référence. La première œuvre de fiction est publiée dans les années quatre-vingts : il s'agit du recueil de nouvelles Vai de Rai Chaze. Dans les années quatre-vingt-dix, paraissent les ouvrages importants signés d'auteurs autochtones, comme Jean-Marc Paubrun, Louise Peltzer, Titaua Peu et Spitz qui seront parfois édités à l'étranger. L'ile des rêves écrasés de Spitz est le premier roman écrit par un Polynésien. 
Ainsi, le fait d'être un écrivain océanien francophone est déjà en soi une forme de transgression : ces écrivains portent la voix de leur peuple, de minorités qui essaient de s'ouvrir à un public plus large, autre que le public insulaire. Le problème de l'insularité est que ce discours peut rester confiné à leur seule île, à un seul territoire. La question est alors de savoir comment passer d'une écriture de l'insularité, de l'ethnicité à une dimension plus universelle. Cette question, Mokaddem la pose dans son essai sur «L'écriture kanake contemporaine »: «Comment, sans affadir la culture kanake, passer d'un seuil géographique délimité par l'insularité vers un autre seuil, qui est celui de la dimension universelle ?» (189). Pourtant, cette parole tente de s'ouvrir à d'autres peuples que ceux de leurs îles, à un autre lectorat, dans cette quête de l'universel. Cette ouverture progressive, « extra-tribale», puis «extra-territoriale » (189) cherche à atteindre un public plus large. Par l'écriture, ces auteurs tentent de transgresser, c'est-àdire de dépasser les frontières, les limites géographiques pour diffuser leur parole, garante du lien culturel de ces territoires d'outre-mer. Pour les uns, il s'agit de trouver son identité dans un paysage multiculturel, pluriethnique; pour les autres, il faut revendiquer son identité, quand le terme même de "Polynésie » est souvent rejeté par la population. Ce sont ces questions, ces dilemmes que nous retrouverons chez ces deux auteures ultra-marines.

\section{Deux femmes libres : des transgressions sous toutes les formes}

Görödé et Spitz sont deux femmes de luttes et de convictions qui ouvrent la voie des non-dits de leur société. Membre du gouvernement chargé de la Culture, de la condition féminine et de la citoyenneté, Görödé n'a jamais cessé d'écrire, comme le prouve la publication en 2012 de son dernier roman, Tâdo, Tâdo, Wéé! ou No more baby. Sa vie est l'incarnation même de la transgression. Licenciée en Lettres modernes, d'abord enseignante, de français puis de paicî, langue vernaculaire de sa tribu, elle se voit incarcérée à trois reprises entre 1974 et 1977 pour ses participations actives dans différentes manifestations indépendantistes. Très engagée politiquement, elle fera partie du mouvement des Foulards rouges, né en 1969, dont elle sera présidente. En 1974, elle crée le « groupe 1878 », à partir de la date de la révolte du grand guerrier Ataï. Puis elle contribue à la création du parti indépendantiste kanak à tendance marxiste, le PALIKA (PArti de LIbération KAnake). Elle participe à l'expérience des Écoles populaires kanak (EPK), créées dans la mouvance des Evénements. Elle est l'une des premières femmes élues à l'assemblée de la Province Nord issues de l'Uni-FLNKS, le parti 
indépendantiste kanak. « Déwé Görödé est une kaapo, celle qui doit rompre les silences pour précisément révéler les visages du non-dit, sur toutes ses latitudes », écrit MarieAnge Somdah (75). Kaapo ou Kaavo est le nom traditionnel de la fille du chef. C'est celle qui peut se permettre d'aller à l'encontre des ordres ou de la tradition. Ainsi, elle met sa plume au service de son peuple, en s'illustrant dans tous les genres : romans, essais, nouvelles, poésie, théâtre. Face au silence (« la Parole des Anciens s’est tue », 31), elle lance un appel déchirant dans son recueil Sous les cendres des conques, en dénonçant cette situation d'exilée sur sa propre terre. Les conques sont de grands coquillages dans lesquels on souffle pour appeler au rassemblement dans les tribus kanak. Les cendres désignent ce qu'il reste des conques, de l'unité kanak, c'est-à-dire « une société fortement éclatée, divisée » («Entretien », 78) après les délimitations des territoires et l'institution des réserves. Cette métaphore désigne donc l'impasse dont l'auteure a pris conscience lors de ses études en métropole. Cependant, cette image peut également être interprétée comme une promesse de renaissance puisque tout le monde sait que les cendres sont fertilisantes. L'écriture a donc une mission sociale : il s'agit de dire la réalité d'un peuple souffrant de vivre dans un pays qui ne leur appartient plus. Ainsi, Görödé manifeste un point de vue de femme kanak engagée dans une lutte politique et féministe. Les titres des regroupements de poèmes sont provocateurs : «Réserves océaniennes » qui fait une référence directe aux Kanak, parqués dans des « réserves » par les colonisateurs ; «Popinées », pour désigner les femmes kanakes ainsi nommées par les blancs. Pour autant, elle n'épargne par ses concitoyens et dénonce également dans son essai Par les temps qui courent les dérives politiques ou les comportements sociaux nuisibles à l'équilibre de tous. Ces apophtegmes sont des maximes visant à corriger ces dérives.

De même, Spitz se donne pour tâche d'écrire ce que les autres n'ont pas dit. Née à Pape'ete (Tahiti), elle a été élevée à l'occidentale dans une famille bourgeoise, formant partie de l'aristocratie «demie » issue des unions entre les descendants des premiers colons et les filles des notables autochtones. Se tournant davantage vers la culture tahitienne qui lui vient de sa famille maternelle, elle choisit de quitter son métier d'institutrice et se révolte contre le système scolaire, notamment sur la question délicate de la colonisation dans l'histoire. Très engagée sur le front culturel, indépendantiste, Spitz participe au mouvement anti-nucléaire né après les premiers essais français de 1966. La question de l'identité tahitienne est un thème récurrent chez elle. La transgression de toutes ces limites va l'amener, en 1991, à écrire son premier roman : L'ile des rêves écrasés. Elle y combat le cliché de la vahiné et des plages de 
sable blanc, symboles de la colonisation, ainsi que les essais nucléaires. Ce roman sera salué par la critique, notamment pour son style qui s'inspire de l'oralité. C'est aussi le premier roman tahitien traduit en anglais (Island of Shattered Dreams). Après un poste de conseillère pédagogique au Ministère de la culture, elle continue de militer après sa retraite contre ce qu'elle nomme un néo-colonialisme insidieux fait de réécriture de l'histoire et de perpétuation d'un mythe, celui qui fige les Tahitiens dans une parodie de «bons sauvages ». En 2001, elle participe, avec d'autres écrivains polynésiens, à la revue littéraire Litterama'ohi, qui publie les textes d'auteurs polynésiens, dans le but de montrer la richesse et la spécificité de ces écrits. S'enchaînent alors d'autres livres, deux romans et un essai, dont le titre est évocateur: Pensées insolentes et inutiles (2006).

Dans son premier roman, L'ile des rêves écrasés, sa mission, celle de réécrire l'histoire de son peuple, peut apparaître derrière les paroles de Tetiare, personnage féminin derrière lequel on croit entendre la voix de l'auteure. Ce personnage déclare : «Tout ce que nous lisons a été écrit par des étrangers. On en arrive presque à croire qu'on est vraiment comme ils nous décrivent, alors que tu sais bien qu'ils n'ont rien compris. Un véritable lavage de cerveau » (162). Ainsi, l'écrivaine postcoloniale se donne pour mission d'être au service d'une cause, celle de son peuple, dont on doit faire entendre la voix. Il semble que l'auteure utilise la double énonciation pour exprimer sa volonté d'écrire, mais également ses doutes et ses inquiétudes: "L'idée est lancée qui coule dans ses veines, sublime et terrifiante : écrire. Se dépouiller pour se donner à l'autre, paroles du rêve pour faire renaître le rêve. Nous rendre à nous-mêmes en retrouvant l'éternité de notre âme. Est-ce que je saurai ?» (162). L'écriture se veut donc neuve, c'est celle d'un rêve, une écriture idéale. Mais le rêve deviendra réalité, lorsque Tetiare, à la fin du roman, parviendra à écrire l'histoire de son peuple et de son pays. Tous les rêves ne sont donc pas « écrasés » car l'écriture devient la mémoire d'un peuple : «Le rêve transmis d'oralité se meurt faute de mémoire et nous devons lui redonner vie par l'écriture. D'autres après toi écriront une parcelle du rêve qui finira par devenir réalité » (201).

Ainsi, les productions des deux auteures s'inscrivent symboliquement dans la thématique de la résistance face au joug colonial français qu'il soit politique, économique ou culturel et donc de la transgression de l'ordre. Elles affirment le droit à l'expression et à l'auto-affirmation car elles représentent un peuple qui se définit comme « colonisé », et dont les aspirations à l'indépendance sont plus que jamais d'actualité. Elles appellent à une renaissance par la lutte, pour retrouver une dignité perdue.

Cet engagement politique se double d'une éthique de la parole : l'oppression des 
minorités est au cœur de leur discours. Dans son roman Hombo, Spitz décrit les hombos, c'est-à-dire les exclus. Le terme rappelle les hobo américains, ces vagabonds un peu clochards qui erraient sur les routes pendant les années de la grande Dépression aux ÉtatsUnis. Des écrivains comme Jack Kerouac s'en sont inspirés dans leurs livres comme On the Road paru en 1957. Spitz les définit comme des « jeunes gens à la lisière de la société, que la société renie. » (83.). Ils n'ont plus aucun rêve, ni aucune joie de vivre. Ils ont été exclus de la population parce qu'ils passent leur temps à boire et à se droguer, et aussi parce qu'ils renient leurs traditions et leurs origines. Ils se réfugient alors dans l'instant, sans se soucier du passé ou de l'avenir. La narratrice dit qu' « ils sont dans une autre dimension dans un non-lieu où la seule façon d'être est le non-être. » (85). Ehu est le héros du livre, ce hombo qui ne connaît plus l'histoire de son pays, et qui vit dans une sorte d'amnésie ou dans « un lent processus de dégradation mémorielle » (144). Comment cette dégradation a-t-elle pu avoir lieu ? Tous les hombos ont refusé de participer aux coutumes et ont renié leurs traditions pour se tourner vers la société moderne. Ils sont devenus des marginaux, c'est-à-dire ceux qui ont transgressé les coutumes et qui se retrouvent exclus de la société. De même, l'œuvre de Görödé présente des personnages victimes de la double oppression coloniale et phallocratique, et s'attache à décrire l'existence de personnages ayant transgressé des interdits. Ainsi, dans son roman Graines de pin colonnaire, l'auteure invite le lecteur à réfléchir sur les malaises de la structure sociale kanak à travers Dany, un travesti. Le jeune homme, qui vit très mal cette identité sexuelle, choisit de la cacher au reste de la famille et de disparaître. Un soir, par hasard, au détour d'une boîte de nuit en ville, la narratrice le retrouve «juché sur de hauts talons fluo [...] dans sa mini-robe moulante » (48). Elle comprend alors pourquoi il a choisi de partir. Implicitement, l'auteure propose une réflexion sur le poids de la coutume, et la « transgression », de l'ordre hétérosexuel.

Dans son roman L'épave, Görödé nous propose une vision de la sexualité très audacieuse. Ainsi, «chacun, homme ou femme, tombe amoureux de quelqu'un qui est interdit : soit par rapport aux convenances sociales coloniales, par exemple la fille du colon de devrait pas aimer le fils du Javanais, soit par rapport aux règles familiales traditionnelles : le fils et la fille d'un même homme ne sauraient tomber amoureux ; c'est une relation taboue. Or le non-dit sur les vrais parents des enfants illégitimes est tel que l'interdit de l'inceste entre frères et sœurs est mis à mal » (Jouve, 2014, communication personnelle). Autrement dit, l'auteure kanak n'hésite pas à transgresser les limites des convenances sociales et à aborder le thème de l'inceste qui nourrit tout le roman. Ces désordres sociaux sont décrits dans une langue très crue qui décrit en détail chaque amour hors-norme, et les transgressions 
orthographiques s'accompagnent d'une grande énergie amoureuse. L'homosexualité féminine kanake y est également révélée au grand jour, puisque les femmes se retrouvent entre elles pour aller au bout de leur jouissance. Éva est cette femme homosexuelle très libre qui a choisi sa vie et qui entretient une relation sexuelle avec Léna, déjà en couple avec Tom. On retrouve l'idée de transgression au sens de dépassement de limites, comme le montre la phrase suivante : «Seules, en situation clandestine et hors-la-loi face à l'univers masculin, leur unique marge résidait dans cette intimité raflée à une vie de soumission à ciel ouvert » (Görödé, Epave, 127). L’homosexualité est ainsi vécue comme la transgression d'un univers à domination masculine et comme un souffle de liberté nouvelle que les deux femmes s'accordent.

\section{La transgression joyeuse}

La transgression, c'est donc l'audace de nouvelles frontières et de nouveaux territoires. En ce sens, elle peut être vécue comme quelque chose de positif, et ainsi rejoindre ce que Foucault affirmait: «Rien n'est négatif dans la transgression. Elle affirme l'être limité, elle affirme cet illimité dans lequel elle bondit, l'ouvrant pour la première fois à l'existence» (238). Ainsi, Foucault tente de redéfinir cette notion en lui conférant un sens cette fois positif. Il faudrait donc enlever à la transgression tout ancrage dans l'éthique, pour la repenser selon la définition bataillienne : ce qui resplendit dans une zone de fêlure, entre le noir et le blanc.

Il est vrai que la transgression des interdits habite nos deux auteures, même la transgression des interdits les plus profonds comme l'inceste chez Görödé. Mais toutes ces transgressions sont mises au service d'un combat en faveur des plus vulnérables, des minorités jusque-là silencieuses. «La colonisation a réuni deux sociétés malades de leurs propres tabous et obsessions », affirme Dominique Jouve. Nos deux auteures ont choisi de se révolter contre les violences infligées aux plus faibles : les femmes, les exclus, les enfants.

Cependant, derrière la lucidité des regards de nos deux auteures, se trouve également une grande liberté d'expression, qui permet de penser que cette transgression peut être qualifiée de «joyeuse ». Leur écriture, qui se fonde sur l'oralité, constitue la transgression majeure de ces œuvres : Görödé retranscrit le verbe kanak, tandis que Spitz retrouve la parole des ancêtres. Elles ne respectent pas les genres occidentaux qu'elles ont empruntés, et mêlent dans leurs œuvres différents discours : ainsi Spitz introduit dans ses romans des passages poétiques, tandis que Görödé décrit les rêves de la narratrice à la fin de son dernier roman Tâdo, Tâdo, wéé ! De même, elles mélangent à la langue française des mots de leurs langues 
natales, dont la plupart ne sont pas traduits au lecteur, comme dans le dernier roman de Spitz, Elles, terre d'enfance, roman à deux encres. On peut relever également de nombreuses constructions agrammaticales chez nos deux auteures quand, par exemple, certains verbes intransitifs deviennent dans leurs livres transitifs directs. Selon Raylene Ramsay, les mots de Görödé sont «'maltraités', 'jetés au dehors', bousculés, comme chez Marguerite Duras, ses lieux communs et expressions toutes faites sont retournés dans tous les sens [...] pour forcer ces mots français à 'dire le vrai', montrer leur dissimulation, leur insuffisance à dire toute la vérité, à dire le silence de la langue maternelle » (148). Ainsi, pour créer un sens nouveau, il faut une langue nouvelle, que l'on va plier à sa guise, jusqu'à ce qu'elle puisse exprimer ce qui était resté innommé. En choisissant la langue de 1'oppresseur, elles transgressent encore les frontières sociales et créent une écriture hybride où le lecteur devra faire un pas vers elles pour comprendre leur message.

\section{Conclusion}

Görödé et Spitz ont donc transgressé toutes les limites, géographiques, historiques, sociales, politiques, discursives, et même la loi du silence. En forçant les mots à « dire le vrai », elles redonnent la parole aux minorités, notamment aux femmes, et proposent une vision très personnelle de ces territoires, loin des clichés paradisiaques véhiculés par les colons. Cette littérature émergente recherche des voies de libération qui demandent d'abord qu'il soit fait table rase des préjugés et limites imposées. Aucun tabou n'existe, et elles explorent même les domaines de l'intimité, en particulier la sexualité, avec une grande puissance. Leur écriture trouve sa force dans cette révolte qui les fait ressembler à Duras. Toutes les conventions langagières sont bannies et le point de vue moralisateur y est absent : au lecteur de se faire sa propre opinion et d'adhérer ou pas à ces propos. Cette forme de littérature transgressive, qui porte les stigmates du passé colonialiste, reste néanmoins joyeuse : la transgression n'est pas régression ou retour vers le passé, mais au contraire c'est une progression. Il convient de " libérer [cette notion] de ce qui est le scandaleux ou le subversif, c'est-à-dire de ce qui est animé par la puissance du négatif » comme l'affirme Foucault (237). Au lecteur de s'interroger.

\section{Bibliographie}

Anova, Apollinaire. Calédonie d'hier, Calédonie d'aujourd'hui, Calédonie de demain. Np : Expressions-Mairie de Moindou, 2005.

Bogliolo, François. Paroles et écritures, anthologie de la littérature néo-calédonienne. Np : 
Hachette Calédonie, 1994 (Entre langues et terre, émergence de la littérature néocalédonienne. Paris : U. Paris III, 2000).

Foucault, Michel. «Préface à la transgression». Dits et écrits, 1954-1988. Tome 1. Paris : Gallimard/NRF, 1994.

Glissant, Édouard. Poétique de la relation. Paris : Gallimard, 1990.

Görödé, Déwé. Sous les cendres des conques. Np : Edipop, 1985.

---. Graines de pin colonnaire. Np : Madrépores, 2009.

---. L'épave. Np : Madrépores, 2007.

---. «Entretien avec Blandine Stefanson ». Notre librairie, revue des littératures du sud 134 (Mai-août 1998).

Mokaddem, Hamid. Littératures calédoniennes, la littérature océanienne francophone estelle une littérature française? La courte échelle. Np : Transit, 1998.

---. «L'écriture kanake contemporaine ». CORAIL. Paris : Harmattan, 1998.

Ramsay, Raylene. «L'hybridité dans l'œuvre de Déwé Görödé : ni bicéphale ni entre-deux ». Littératures d'émergence et mondialisation, actes du colloque UNC 2003. Np: In Press 2004.

Somdah, Marie-Ange. « Déwé Görödé ou la recherche de la Parole kanak ». Notre librairie, revue des littératures du sud 134 (Mai-août 1998).

Soula, Virginie. Histoire littéraire de la Nouvelle-Calédonie. Np : Karthala, 2014.

Spitz, Chantal. Litterama'ohi 16 (juin 2009).

---. L'ile des rêves écrasés. Np : Au vent des îles, 2007.

---. Hombo. Np : Au vent des îles, 2012.

Sultan, Patrick. La scène littéraire postcoloniale / L'esprit des Lettres. Np : Manuscrit, 2011. 